\title{
Creencias y actitudes de los jóvenes universitarios chilenos hacia las variedades cultas del español
}

\author{
Silvana Guerrero* \\ Abelardo San Martín \\ Universidad de Chile
}

\begin{abstract}
Resumen
En este trabajo se informan los resultados de una investigación sobre las percepciones de jóvenes universitarios chilenos, tanto hacia variedades diferentes del español como hacia su propia variedad, en el marco del PRECAVES XXI. El objetivo de este estudio es determinar las actitudes lingüísticas de 100 de estos informantes, en términos de qué variedades consideran como mejores y más prestigiosas y qué percepciones tienen de su propia variedad de español, atendiendo a valoraciones directas (cognitivas y afectivas) e indirectas. Se aplicó una encuesta que combina los métodos directo e indirecto de recogida de datos a través de la técnica de pares falsos (matches guise). Los principales hallazgos de este estudio indican que, para la mayoría de los sujetos de la muestra, no existe una variedad de español mejor que otra y, entre quienes se decantan por alguna, las variedades andina y chilena cuentan con mayor prestigio. Asimismo, existen actitudes
\end{abstract}

\footnotetext{
Para correspondencia, dirigirse a: Silvana Guerrero González (siguerrero@u.uchile.cl) o Abelardo San Martín Núñez (asmartin@uchile.cl), Departamento de Lingüística, Facultad de Filosofía y Humanidades, Universidad de Chile, Ignacio Carrera Pinto 1025, Tercer Piso, Ñuñoa, Santiago, Chile.
} 
directas e indirectas muy positivas hacia la variedad chilena. Por último, los informantes tienden a valorar positiva y negativamente aspectos fonéticos de otras variedades del español.

Palabras clave: creencias lingüísticas, actitudes lingüísticas, variedades del español, PRECAVES XXI, español de Chile.

Recibido: $14 / 02 / 18$

Aceptado: 17/04/18

\title{
Beliefs ANd ATtitudes of young ChILEAN University STUdENTS TOWARDS THE EDUCATED VARIETIES OF SPANISH
}

\begin{abstract}
In PRECAVES XXI context, this paper we report the results of a research on the perceptions of young Chilean university students, both to different Spanish and to their own variety. The aim of this study is to analyze the linguistic attitudes of 100 of these subjects, in terms of which varieties they consider to be better and more prestigious and what perceptions they have of their own variety of Spanish, attending to direct evaluations (cognitive and affective) and indirect. A survey was conducted combining direct and indirect methods of data collection using the matches guise technique. The main findings of this study indicate that, for most of the subjects in the sample, there is not one variety of Spanish better than another, and among those who prefer one, the Andean and Chilean varieties have more prestige. Likewise, there are direct and indirect attitudes very positive towards the Chilean variety. Finally, in general, informants value positively and negatively phonetic aspects of other varieties of Spanish.
\end{abstract}

Keywords: linguistic beliefs, linguistic attitudes, Spanish varieties, PRECAVES XXI.

\section{INTRODUCCIÓN}

Las creencias y las actitudes de los hablantes son un factor determinante en la particular configuración de la variación sociolingüística de una comunidad de habla (Labov 1983). Estas respuestas cognitivas o afectivas hacia el lenguaje están en la base de la valoración positiva (prestigio) o 
negativa (estigmatización) del uso lingüístico; por lo tanto, constituyen un factor explicativo relevante de dicha variación (Moreno Fernández 2009a y López Morales 2015). No obstante, a pesar de su importancia, aún no se ha dado la suficiente cabida a los estudios sobre actitudes lingüísticas en el medio hispánico. Solo en el último tiempo se han gestado iniciativas de alcance panhispánico para su estudio. En el marco del Proyecto para el Estudio de Creencias y Actitudes hacia las Variedades del Español en el siglo XXI (PRECAVES XXI) (Cestero y Paredes 2015a, 2015b y 2018 - este último, en este mismo monográfico-; Paredes y Cestero 2018), el objetivo de este trabajo es analizar las creencias y las actitudes lingüísticas de 100 hablantes jóvenes chilenos universitarios, tanto hacia variedades diferentes del español como hacia su propia variedad de habla. Las preguntas que orientan esta investigación son a) ¿qué variedad o variedades consideran los jóvenes universitarios chilenos como mejores y más prestigiosas?, b) ¿qué percepción tienen estos sujetos de su propia variedad? y c) ¿en qué medida identifican el resto de las variedades cultas del español? Específicamente, en este estudio se pretende: a) determinar qué variedad o variedades del español consideran los sujetos como mejores o más prestigiosas, b) describir sus percepciones sobre la variedad de habla chilena y c) señalar el grado en que identifican otras variedades cultas del español. Para este último propósito, se estudian las valoraciones directas (cognitivas y afectivas) e indirectas (características sociales de la persona que habla, características de la región y características de la cultura) de los informantes, en relación con la voz que evalúan (femenina o masculina), su sexo (mujer u hombre) y su conocimiento acerca de las variedades del español (sin o con estudios sobre dicho tema). Se incluyen, asimismo, algunos datos generales acerca de la percepción que tienen los jóvenes universitarios chilenos sobre otras variedades cultas del español, en términos de: a) reconocimiento de la variedad que escuchan y b) aspectos de cada variedad valorados positiva y negativamente.

\section{MARCO TEÓRICO}

Las actitudes lingüísticas consisten en percepciones o valoraciones subjetivas de los hablantes hacia el uso lingüístico, ya sea que se refieran a un fenómeno, en particular, o a una variedad de lengua o lengua, en general (Blas Arroyo 2005). Huelga advertir que el concepto de "actitud" surgió en la psicología social y, con posterioridad, se aplicó en el ámbito de la sociología del lenguaje y la sociolingüística. En dichos dominios se ha generado cierto acuerdo en 
que las actitudes se pueden analizar en tres tipos de componente: cognitivo, afectivo, conativo. El primero está relacionado con una creencia, que incide en un criterio de corrección lingüística; el segundo implica una valoración, que puede medirse en términos del agrado que se provoca en el hablante y el tercero involucra una conducta o reacción (Moreno Fernández 2009a). Asimismo, existe consenso en destacar la importancia de las actitudes lingüísticas para los análisis de la sociolingüística y la sociología del lenguaje, ya que dichas percepciones inciden de manera estratégica en el comportamiento de los hablantes, con el propósito de promover los procesos de cambio lingüístico específicos o imprimir mayor vitalidad a los dialectos o las lenguas en situaciones de bidialectalismo o bilingüismo (Moreno Fernández 2009a, Trudgill y Hernández Campoy 2007, López Morales 2015, García Marcos 2015 y Silva-Corvalán y Enrique-Arias 2017). En particular, las actitudes lingüísticas pueden ser muy relevantes en la determinación de las comunidades de habla (Labov 1983, Blas Arroyo 1994 y 2005) y en la medición de la conciencia lingüística de los hablantes (López Morales 2015).

En otro orden, existen dos enfoques principales en el estudio de las actitudes lingüísticas: el conductista y el mentalista. De acuerdo con el primero, se analizan las opiniones declaradas por los hablantes sobre el uso lingüístico; para el segundo, las actitudes corresponden más bien a un estado mental interior de los sujetos que media entre un estímulo y su respuesta (Blas Arroyo 2005). En primer enfoque propicia la aplicación de métodos directos para el estudio de las actitudes que consisten en la declaración manifiesta de las posturas de los individuos hacia determinadas preguntas. El enfoque mentalista, en cambio, implica la articulación de métodos indirectos que indaguen sobre los procesos de introspección interna de los hablantes como, por ejemplo, la técnica de pares falsos (matches guise), en la que se emplean grabaciones y cuestionarios de pares ocultos (Blas Arroyo 2005, Silva-Corvalán y Enrique-Arias 2017). En la actualidad, algunos autores han insistido en la necesidad de combinar los métodos directos e indirectos en la indagación de las actitudes lingüísticas, a efecto de neutralizar, en parte, los sesgos atribuidos a cada método por separado (Rojas 2012a).

Una de las líneas de investigación de interés en el estudio de las actitudes lingüísticas consiste en indagar las que los hablantes dispensan hacia lenguas y variedades de lengua (dialectos, por ejemplo) diferentes. De hecho, este es uno de los focos de interés que se han desarrollado en el último tiempo y que han justificado iniciativas de conjunto como el proyecto Linguistics Identity and Attitude in Spanish Speaking Latin America (LIAS) y el PRECAVES XXI. En Cestero y Paredes (2015a, 2015b) y Paredes y Cestero (en prensa) los gestores de este último proyecto informan resultados preliminares de su 
estudio de las actitudes de hablantes de la comunidad de Madrid, además de perfilar los lineamientos generales de la metodología del PRECAVES XXI. Según estos autores, en general, los sujetos madrileños manifiestan actitudes positivas hacia todas las variedades normativas de español consideradas, aunque la variedad mejor valorada es la propia, mientras la andaluza es la que obtiene la apreciación más baja. Asimismo, el componente afectivo es mejor evaluado que el cognitivo y ambos componentes están correlacionados con las percepciones de las variedades relevadas.

Por lo que refiere a la indagación de las actitudes de hablantes chilenos hacia las variedades geográficas del español, contamos con los estudios de Rojas (2012a, 2012b, 2012c, 2013), quien emplea una encuesta de método directo aplicada a una muestra de 400 hablantes santiaguinos, en el marco del proyecto LIAS. Este autor se refiere en los siguientes términos a la importancia de la investigación de las actitudes lingüísticas: "el estudio de las actitudes lingüísticas en la actualidad resulta de interés transversal para variados campos de estudio dentro de las disciplinas que deben enfrentarse con problemas en que los aspectos sociales afectan al uso del lenguaje" (Rojas 2012a: 42). Los resultados de sus estudios revelan que, en cuanto a las dimensiones de corrección y agrado hacia variedades geográficas del español, existe una coincidencia entre las variedades más correctas y las más agradables, a saber, el español de España, el de Perú y el de Colombia. El rasgo que caracteriza a estas variedades es su consonantismo conservador, el que es muy diferente al del español de Chile; por lo tanto, para Rojas el nivel fonético es el más saliente en la determinación de las actitudes de los hablantes chilenos. Por otro lado, a pesar de que el español de Perú alcanza un alto nivel de preferencias por parte de los sujetos de la muestra, Rojas interpreta que la variedad más correcta para los chilenos es el español de España, debido a baja valoración social del grupo inmigrante peruano. La excepción a esta coincidencia entre corrección y agrado en su indagación es el español de Chile que es una de las más agradables para los sujetos, pero al mismo tiempo es calificada como la menos correcta de las variedades. De acuerdo con Rojas habría una diferencia de tipo de prestigio entre las variedades de España y Chile: un prestigio abierto para el español peninsular y uno encubierto para el chileno, aspecto que podría explicar esta inconsistencia entre las dimensiones de agrado y corrección respecto de la propia variedad de habla de los hablantes chilenos. 


\section{METODOLOGÍA}

En este estudio se ha adoptado la metodología general del PRECAVES XXI. Este último consiste en una iniciativa de alcance panhispánico que se propone indagar las actitudes hacia las variedades de la lengua española mediante la técnica de encuesta basada en pares falsos. Asimismo, el mencionado instrumento permite que los sujetos encuestados evalúen muestras reales de lengua hablada (Cestero y Paredes 2015a, 2015b, 2018).

\subsection{Diseño DE LA ENCUESTA}

La encuesta realizada aúna métodos directos e indirectos de recogida de datos a través de la técnica de pares falsos. La aplicación del instrumento, cuya duración es de una hora aproximadamente, está dividida en tres partes: a) recogida de datos personales y sociodemográficos del informante, $b$ ) escucha de grabaciones y c) realización de un cuestionario.

\subsection{LOS INFORMANTES}

Los datos personales que son incluidos en PRECAVES XXI, por su posible incidencia en las creencias y actitudes de los hablantes hacia las variedades del español, son sexo, edad, nivel de estudios, profesión y lengua materna, país de nacimiento y países hispanohablantes visitados por el informante. Se incluye, además, información sobre su uso de Internet y de televisión. En cuanto a aquellos sujetos con estudios universitarios en el área de lengua y literatura, es pertinente distinguir si cuentan con formación en variedades del español o no.

La tabla 1 muestra la distribución de los informantes que contestaron la encuesta para el presente trabajo. 


\begin{tabular}{llcc} 
& & Cantidad & Porcentaje \\
\cline { 3 - 4 } Sexo $^{1}$ & Hombre & 38 & $38 \%$ \\
& Mujer & 62 & $62 \%$ \\
\hline Nacionalidad & Chilena & 100 & $100 \%$ \\
\hline Edad & Menos de 20 (entre 18 y 19 años) & 23 & $23 \%$ \\
& Entre 20 y 34 & 77 & $77 \%$ \\
\hline Nivel de & Universitarios & 100 & $100 \%$ \\
estudios & (Estudiantes de Lengua y Literatura & & \\
& Hispánica o Inglesa) & & \\
\hline Colectivo & Sin formación en variedades & 50 & $50 \%$ \\
& Con formación en variedades & 50 & $50 \%$ \\
\hline
\end{tabular}

Tabla 1. Distribución de los informantes que conforman la muestra

\subsection{LAS GRABACIONES}

La recogida de materiales está basada en la escucha de 16 grabaciones, 8 correspondientes a discurso oral y 8 a lectura de texto escrito, de hombres y mujeres adultos de entre 34 y 54 años, con nivel de estudios superiores. Las grabaciones de voces masculinas y de voces femeninas pertenecen a las 8 variedades normativas del español, según Moreno Fernández (2009b), a saber, castellana, andaluza, canaria, mexicana-centroamericana, caribeña, andina, chilena y rioplatense.

\subsection{El CUESTIONARIO}

El cuestionario utilizado para la recolección de los datos incluye preguntas directas e indirectas, abiertas y cerradas, en la mayor parte de los casos, con escala de diferencial semántico. A través de estas preguntas se pretende obtener información sobre las valoraciones y las actitudes lingüísticas de los hablantes chilenos hacia diferentes variedades del español, incluido 
el español de Chile. Las preguntas del cuestionario se dividen en cuatro dimensiones: a) valoración de la variedad o las variedades que corresponden al mejor español, b) valoración directa de la propia variedad de habla, c) valoración indirecta de dicha variedad a través de la persona que habla y d) valoración indirecta de esa variedad mediante la región y la cultura.

\section{RESULTADOS}

Como hemos anticipado, para orientar nuestra búsqueda nos propusimos responder a las preguntas sobre qué variedades consideran los sujetos como mejores y más prestigiosas y qué percepciones tienen de su propia variedad de habla, en cuanto a los aspectos valorados positiva y negativamente. Para esto, hemos identificado, en primer lugar, la valoración sobre lo que consideran 'mejor español' y, luego, nos hemos enfocado en aquellos casos en los que los informantes reconocen de manera correcta la variedad chilena. Para tal cometido, contamos con los resultados de una encuesta que combina los métodos directo e indirecto de recogida de datos, a través de la técnica de pares falsos (matches guise).

Para comenzar, informaremos los resultados de nuestro estudio acerca de las percepciones de los sujetos de la muestra sobre las variedades del español en general, esto es, qué variedad o variedades son consideradas por los informantes como mejores o de mayor prestigio. En segundo término, nos referiremos a las percepciones directas e indirectas de los sujetos acerca de la variedad de habla chilena, es decir, qué valoraciones (positivas o negativas) manifiestan hacia su propio dialecto. En términos estadísticos recurriremos, la mayor parte de las veces, a descripciones basadas en porcentajes, aunque también daremos cuenta de la aplicación de pruebas estadísticas (Chi cuadrado y ANOVA).

Por último, se incluyen algunos datos generales sobre la percepción que tienen los jóvenes universitarios chilenos sobre otras variedades del español, en particular, con respecto al reconocimiento de las variedades y a los aspectos de cada una valorados positiva y negativamente.

\subsection{Percepciones sobre Las variedades del español en General}

En el gráfico 1 exponemos los resultados de nuestra indagación sobre la variedad de español mejor evaluada por los sujetos de la muestra. 


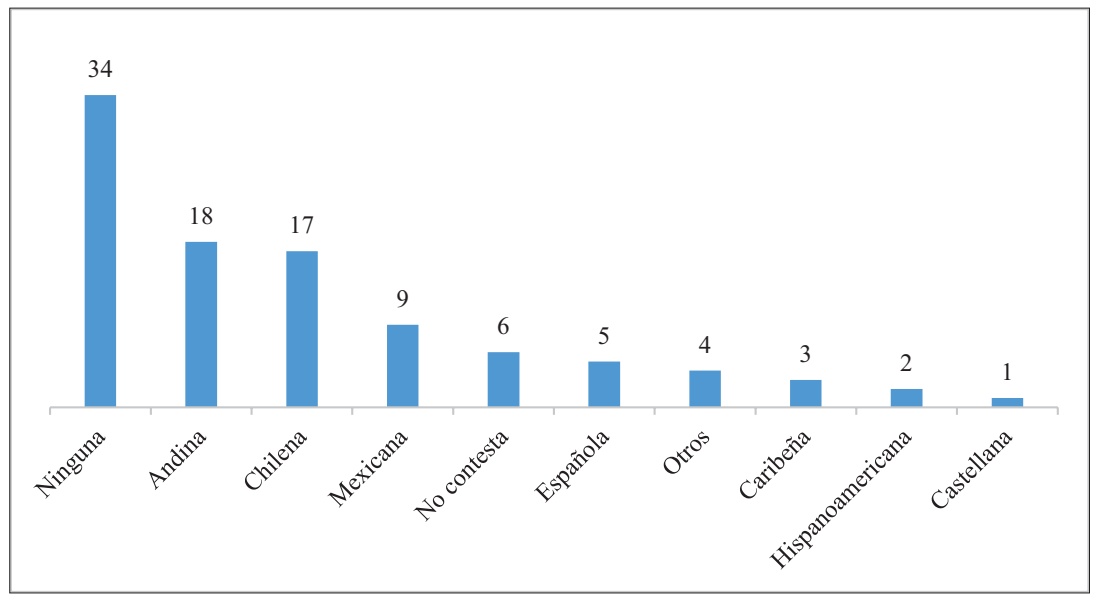

Gráfico 1. Valoración sobre mejor español (porcentajes)

En lo que respecta al análisis de la variedad o variedades que se consideran como más prestigiosas, los informantes optan por señalar que ninguna es más prestigiosa que otra, llegando incluso a explicitar que se trata solo de variedades o que "No hay una región que hable mejor, solo se habla diferente". Esta opción es la preferida por 34 sujetos. En orden de frecuencia, le sigue la variedad andina, seleccionada en 18 ocasiones como la variedad de mejor español que en las opiniones de los encuestados, en la mayoría de los casos, está representada por el dialecto de Perú. Recordemos que esta variedad es también valorada muy positivamente por los informantes de los estudios de Rojas (2012a, b y c). En tercer lugar, los sujetos eligen la variedad chilena en 17 casos, lo que podría estar relacionado con la positiva percepción que tienen los informantes sobre su propia variedad dialectal. Con frecuencias muy bajas se eligen la variedad mexicana $(9 / 100,9 \%)$, la española (5\%), otros (4\%), en cuyo caso se alude a "las capitales" o "zona centro", en general, la caribeña e hispanoamericana ( $3 \%$ cada una) y, por último, la variedad castellana con apenas 1 caso. La aplicación de pruebas no paramétricas revela que estos datos son significativos estadísticamente $($ Chi cuadrado $=77,800 ; p=0,000)$.

Algunas de las opiniones de los encuestados acerca de las variedades son las siguientes: "a mi juicio, no existe un ideal del idioma" $\left(324^{2}\right)$, "no

\footnotetext{
2 El número a continuación de cada ejemplo corresponde al código del sujeto en la muestra del PRECAVES XXI.
} 
creo que haya una forma de español mejor que otra" (332), "no considero que en un lugar u otro se hable mejor o peor (335)", "en ninguna se habla mejor que en otra" (336), "no considero que el español se hable mejor en una zona u otra. Sin embargo, la variante chilena me suena más cómoda y clara que las variantes de otros países" (740) y "son simplemente distintas variedades, no hay ninguna que sea 'mejor' o 'peor' que otra" (1141). El contraste entre el colectivo de estudiantes sin formación en variedades del español y el que sí la tiene no arroja diferencias respecto de la percepción sobre la mejor variedad de esta lengua.

\section{2. Percepciones Sobre La VARIEdAd Chilena en Particular}

En relación con la identificación de la variedad propia por parte de los informantes, de los 100 informantes, el 95\% responde de manera acertada, es decir, en el total de casos en que los sujetos percibieron la variedad chilena en la voz masculina o en la voz femenina, la mayoría la identificó como propia. El 4\% la percibió como mexicana y el 1\% como caribeña. No obstante, hubo 12 casos en que los sujetos, además de identificar correctamente la variedad chilena, señalaron como propia la variedad canaria. Asimismo, en 2 ocasiones los informantes también reconocen como propia la variedad mexicana y en 1 oportunidad reconocen como propia la variedad andaluza y castellana, además de la chilena. Estos datos se presentan en la tabla siguiente.

\begin{tabular}{llc}
\hline Grabaciones valoradas (N=100) & Frecuencia & Porcentaje \\
\hline $\begin{array}{l}\text { Variedad identificada de manera } \\
\text { correcta }\end{array}$ & 95 & $95 \%$ \\
Variedad identificada de manera & $4=$ variedad & $4 \%$ \\
incorrecta & $\begin{array}{l}\text { mexicana } \\
1=\text { caribeña }\end{array}$ & $1 \%$ \\
Variedad identificada de manera & $12=$ variedad & $12 \%$ \\
correcta, pero incluyendo otras & canaria & $4 \%$ \\
variedades como propias & $4=$ variedad & $2 \%$ \\
& andaluza & $1 \%$ \\
& $2=$ variedad & \\
& mexicana & \\
& $1=$ variedad & \\
& castellana & \\
\hline
\end{tabular}

Tabla 2. Identificación acertada y errónea de la propia variedad 
Respecto de la valoración de la variedad como similar o diferente, la mayor parte de los informantes la reconoce como idéntica o similar (91\%) y el $9 \%$ restante la identifica como no idéntica. De manera específica, los datos proporcionados por los sujetos encuestados se incluyen en la tabla 3.

\begin{tabular}{lcc}
\cline { 2 - 3 } & $\begin{array}{l}\text { Informantes chilenos (N=100. } \\
\text { Cada sujeto evalúa 2 voces= } \\
\text { oral y escrita =200) }\end{array}$ & Porcentaje \\
\hline No idéntica o similar & $\mathbf{1 8}$ & $\mathbf{9 \%}$ \\
Totalmente diferente & 5 & $2,5 \%$ \\
Bastante diferente & 4 & $2 \%$ \\
Ligeramente diferente & 9 & $4,5 \%$ \\
Idéntica o similar & $\mathbf{1 8 2}$ & $\mathbf{9 1 \%}$ \\
Ligeramente igual & 10 & $5 \%$ \\
Bastante igual & 42 & $21 \%$ \\
Totalmente idéntica & 130 & $65 \%$ \\
\hline
\end{tabular}

Tabla 3. Proporción sobre similitud y diferencia

Por otro lado, en el gráfico 2 se presenta el desglose de los aspectos que son valorados positivamente por parte de los informantes sobre la variedad chilena.

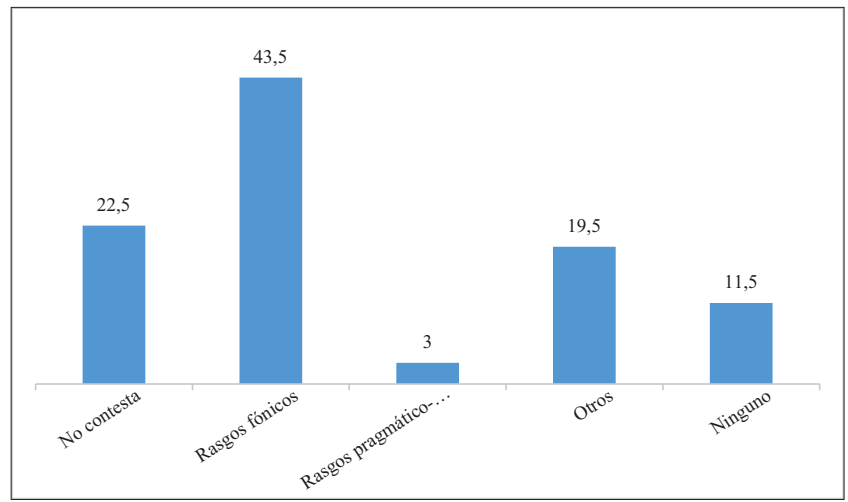

Gráfico 2. Aspectos valorados positivamente (porcentajes)

Del total de aspectos valorados por audio escuchado, son los rasgos fónicos los que presentan la mayor aceptación positiva. Se trata, en concreto, de 87 $(43,5 \%)$ casos en los que los informantes identifican algún aspecto fónico de 
la voz evaluada como positivo. Destacan el acento, la entonación, la fluidez y el ritmo como aquellas características que identifican de manera positiva, aunque la mayoría de las veces no se señalan rasgos fonéticos concretos. En el trabajo de Rojas (2012a), el nivel fonético es también el más destacado en la determinación de las actitudes de los hablantes chilenos. En particular, los encuestados en dicho estudio aluden a la retención de consonantes como /-s/ y /-d-/ como rasgos valorados positivamente de las variedades peruana y española; en cambio, su pérdida es evaluada de modo negativo en la variedad chilena. Por lo tanto, nos parece coherente que en nuestro trabajo se señalen como rasgos fonéticos positivos de la variedad chilena categorías más bien generales como el acento y la entonación, por ejemplo. Luego, en 45 $(22,5 \%)$ oportunidades los informantes no responden. En la categoría otros, que es identificada en 39 (19,5\%) ocasiones, son relevantes aspectos como lo bonita, lo alegre, lo sencilla y lo agradable de la voz escuchada. Además, la categoría ninguno se registró en $23(11,5 \%)$ oportunidades. Los rasgos pragmático-discursivos son identificados en apenas $6(3 \%)$ oportunidades y suele tratarse de las "muletillas".

El gráfico 3 expone los aspectos peor valorados de la variedad chilena.

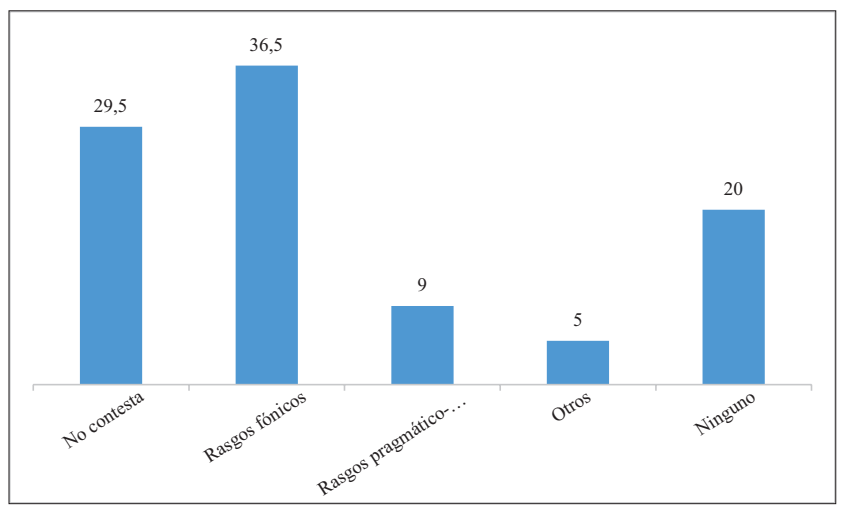

Gráfico 3. Aspectos valorados negativamente (porcentajes)

También son los rasgos fónicos aquellos que presentan la frecuencia más elevada, esto es, en 73 ocasiones (36,5\%) los informantes reconocen algún aspecto fónico como aquel que es característico de una actitud negativa hacia la variedad. A diferencia de los aspectos mejor valorados, donde destacan rasgos como la fluidez y el ritmo de la variedad, en este caso son destacables lo monótono, que suele relacionarse con los casos en que se escuchó la lectura de un texto; la rapidez o la lentitud del habla, la pronunciación incompleta 
de algunas palabras y la pronunciación de algunos fonemas concretos, como la $j$, la $c h$, la $y$, la $g$ u otros declarados por los informantes. Cabe destacar que en 59 oportunidades $(29,5 \%)$ los sujetos de la muestra no contestan. La categoría "ninguno" es identificada en $40(20 \%)$ ocasiones y con ella los sujetos se refieren a aspectos como lo distante, lo feo y lo desagradable de la voz evaluada, es decir, de los rasgos opuestos a los valorados positivamente. Con una frecuencia muy baja aparecen los rasgos pragmático-discursivos (18, $9 \%$ ), donde se identifican, sobre todo, la repetición de palabras, las muletillas y las vacilaciones de los hablantes. Por último, aparece la categoría "otros" con apenas 10 casos $(5 \%)$.

\subsubsection{Valoraciones directas de la variedad chilena}

En el gráfico 4 se ordenan los datos correspondientes a las valoraciones directas de la variedad chilena, considerando las medias según las dimensiones cognitiva, afectiva y general de los sujetos que contestaron la encuesta.

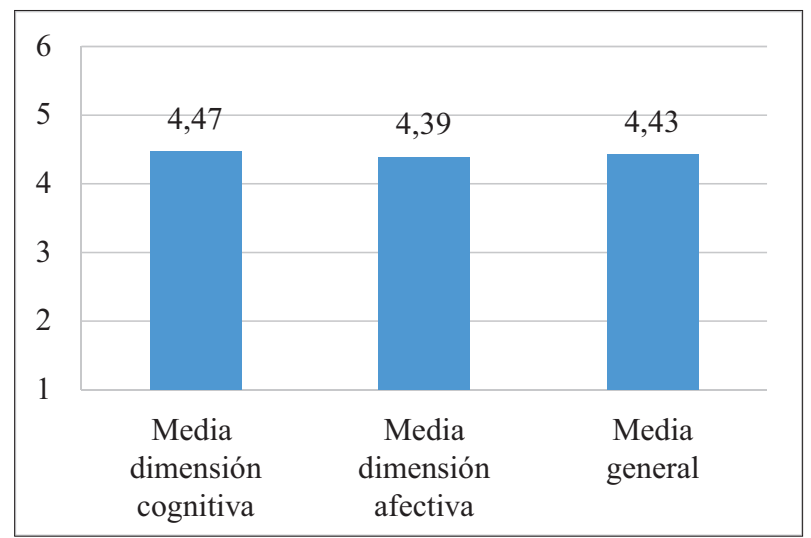

Gráfico 4. Medias según las dimensiones cognitiva, afectiva y general

El gráfico que precede permite apreciar que prácticamente no existen diferencias entre las medias de la dimensión cognitiva y las de la dimensión afectiva. Los aspectos cognitivos, sin embargo, son valorados más positivamente. Los datos correspondientes a cada dimensión se presentan en los gráficos 5 y 6 . 


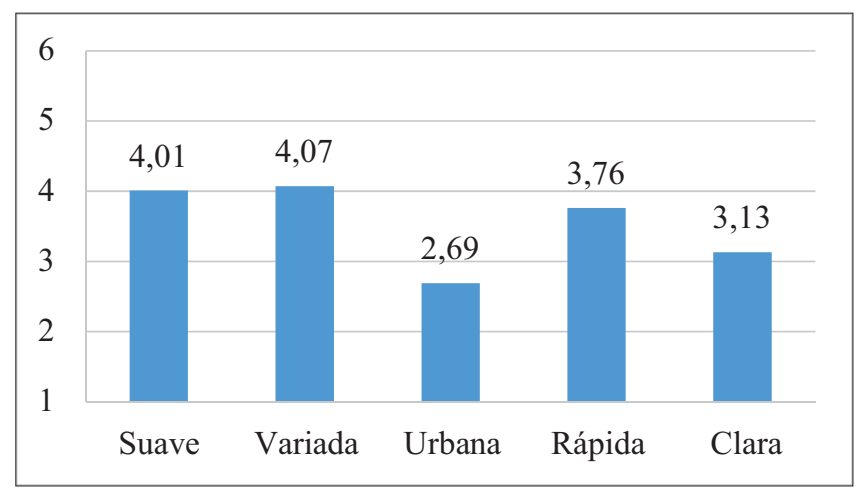

Gráfico 5. Medias de valoración cognitiva

Como se observa en el gráfico 5, es la media urbano-rural la que presenta la evaluación más baja. Los aspectos vinculados con lo suave (por oposición a lo áspera) o lo variada (opuesto a monótona) de la voz evaluada representan las medias más altas. Finalmente, presentan medias similares los aspectos rapidez (en oposición a lentitud) y claridad (opuesto a confusa).

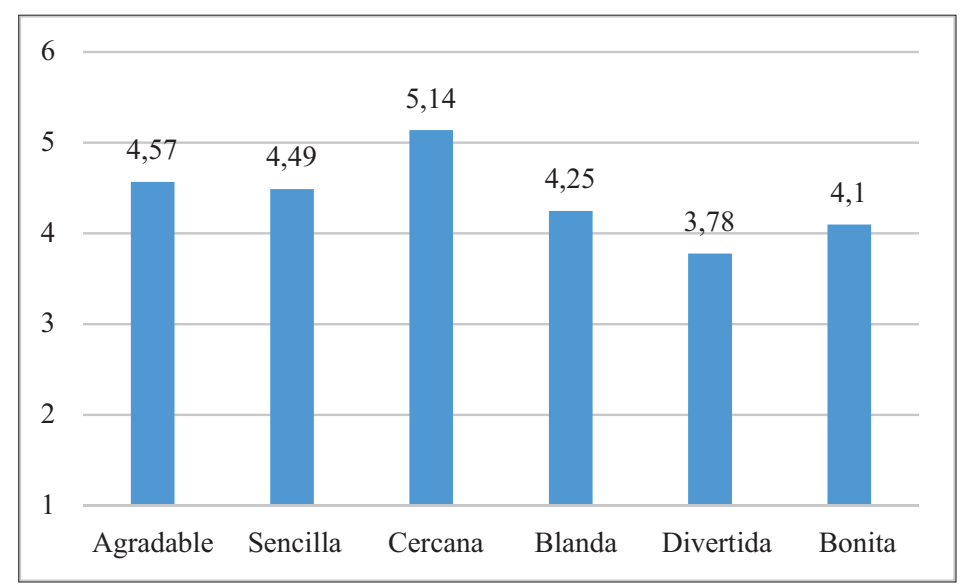

Gráfico 6. Medias de valoración afectiva

Los datos obtenidos para la valoración de la dimensión afectiva nos permiten precisar que los promedios son bastante homogéneos. El único caso que supera los 5 puntos es la cercanía (opuesto a distante) de la voz evaluada, y solo lo divertida (opuesto a aburrida) baja de los 4 puntos para la dimensión en estudio. El resto de las medias se ordena sobre los 4 puntos y muestra que 
hay una valoración muy positiva de la propia variedad de habla por parte de los sujetos de la muestra.

La tabla 4 expone los resultados del cruce entre las valoraciones directas y el tipo de voz evaluada, el sexo y el colectivo del informante.

\begin{tabular}{|c|c|c|c|c|c|c|c|c|c|}
\hline & $\begin{array}{c}\text { Media } \\
\text { cognitiva }\end{array}$ & $\mathrm{F}$ & $\mathrm{p}$ & $\begin{array}{c}\text { Media } \\
\text { afectiva }\end{array}$ & $\mathrm{F}$ & $\mathrm{p}$ & $\begin{array}{c}\text { Media } \\
\text { general }\end{array}$ & $\mathrm{F}$ & $\mathrm{p}$ \\
\hline Voz masculina & 4,215 & \multirow[t]{2}{*}{34,082} & \multirow[t]{2}{*}{0,000} & 4,289 & \multirow[t]{2}{*}{0,927} & \multirow[t]{2}{*}{0,337} & 4,252 & \multirow[t]{2}{*}{19,567} & \multirow[t]{2}{*}{0,000} \\
\hline Voz femenina & 4,829 & & & 4,402 & & & 4,615 & & \\
\hline Hombre & 4,247 & \multirow[t]{2}{*}{12,322} & \multirow[t]{2}{*}{0,001} & 4,322 & \multirow[t]{2}{*}{0,58} & \multirow[t]{2}{*}{0,810} & 4,284 & \multirow[t]{2}{*}{6,003} & \multirow[t]{2}{*}{0,015} \\
\hline Mujer & 4,645 & & & 4,351 & & & 4,498 & & \\
\hline Sin formación & 4,562 & \multirow[t]{2}{*}{1,495} & \multirow[t]{2}{*}{0,223} & 4,307 & \multirow[t]{2}{*}{0,324} & \multirow[t]{2}{*}{0,570} & 4,434 & \multirow[t]{2}{*}{0,172} & \multirow[t]{2}{*}{0,679} \\
\hline $\begin{array}{l}\text { Con } \\
\text { formación }\end{array}$ & 4,424 & & & 4,374 & & & 4,399 & & \\
\hline
\end{tabular}

Tabla 4. Dimensiones cognitiva y afectiva según voz evaluada, sexo y colectivo

Las medias de las dimensiones cognitiva, afectiva y general son más altas en el caso de la voz evaluada femenina y de las informantes mujeres. La aplicación de la prueba ANOVA, además, indica que, respecto del factor sexo, se trata de diferencias con significación estadística. También son más altas las medias de las valoraciones de los sujetos sin formación en variedades del español, en cuanto a los aspectos cognitivo y general, aunque no por lo que refiere a la dimensión afectiva; se trata, sin embargo, de datos que estadísticamente no fueron significativos.

Otros hallazgos sugieren que los hombres diferencian más al evaluar voces femeninas y masculinas, pues, en el caso de las mujeres, al evaluar ambas voces, las medias son similares (3,3 al evaluar a mujeres y 3,4 al evaluar voces masculinas), mientras que las de los hombres se distancian, y las medias de los hombres al evaluar voces femeninas son más altas que las del resto de los grupos (3,6 al evaluar voces femeninas y 3,2 al evaluar voces masculinas). En este sentido, los hombres evaluando a mujeres y las mujeres evaluando a mujeres se comportan de manera diferente. En la dimensión afectiva, los hombres valoran más positivamente las voces femeninas y las mujeres evalúan mejor las voces masculinas, pero son los informantes hombres quienes diferencian más ambas voces evaluadas.

Se puede precisar que los datos de la media general no solo están determinados por la valoración directa afectiva, sino que, además, se relacionan con la valoración positiva de los hombres evaluando voces femeninas. 


\subsubsection{Valoraciones indirectas de la variedad chilena}

Los datos correspondientes a las valoraciones indirectas de la variedad chilena a través de la persona que habla, específicamente, sobre las características sociales, se incluyen en la tabla 5.

\begin{tabular}{lcc}
\hline & $\begin{array}{c}\text { Frecuencia }(\mathrm{N}=100 . \text { Cada } \\
\text { sujeto evalúa 2 voces= } \\
\text { oral y escrita =200) }\end{array}$ & Porcentaje \\
\hline Trabajo & & \\
No contesta & 3 & $1,5 \%$ \\
Poco cualificado & 14 & $7 \%$ \\
Bien cualificado & 116 & $58 \%$ \\
Altamente & 67 & $33,5 \%$ \\
cualificado & & \\
Ingresos & & $1,5 \%$ \\
No contesta & 3 & $3 \%$ \\
Bajo & 6 & $55,5 \%$ \\
Medio & 111 & $40 \%$ \\
Alto & 80 & $1,5 \%$ \\
Estudios & & $0,5 \%$ \\
No contesta & 3 & $2 \%$ \\
Sin estudios & 1 & $21,5 \%$ \\
Primarios & 4 & $74,5 \%$ \\
Secundarios & 43 & \\
Universitarios & 149 & \\
\hline
\end{tabular}

Tabla 5. Valoraciones indirectas de la variedad chilena a través de la persona que habla: características sociales

Como se aprecia en la tabla precedente, los encuestados, en su mayoría, coincidieron en las calificaciones que hicieron respecto de las voces que evaluaban. La mayoría de ellos identificó las voces como aquellas propias de personas con trabajos bien cualificados, con ingresos medios y con estudios universitarios. Estas características representan efectivamente a las voces evaluadas que, además, constituyen voces muy representativas de la comunidad de habla en estudio. Las medias obtenidas para las valoraciones indirectas de la variedad chilena a través de la persona que habla se exponen en el gráfico 7. 


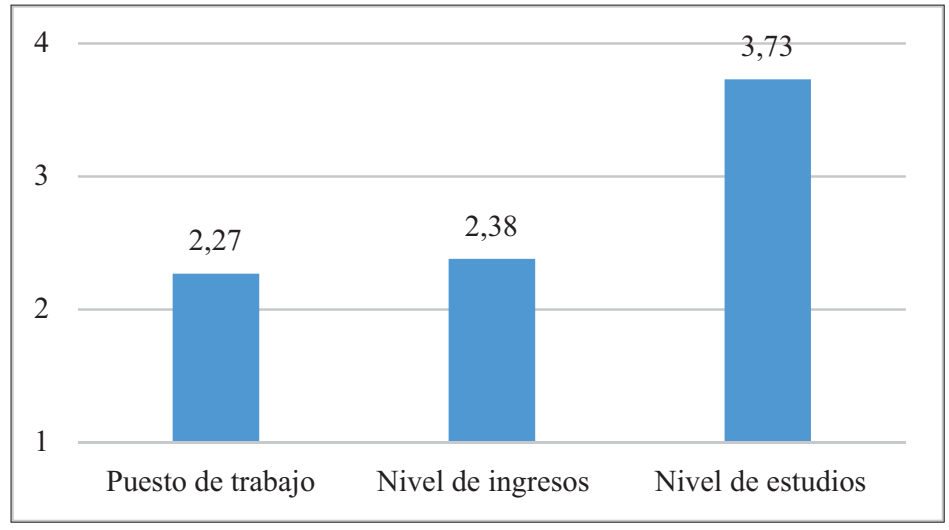

Gráfico 7. Valoraciones indirectas de la variedad chilena a través de la persona que habla

Las medias del gráfico 7 permiten corroborar que es el nivel de estudios la dimensión que es valorada más positivamente por los sujetos que respondieron la encuesta, es decir, para los encuestados, este es el factor determinante en la cualificación de una persona. Las medias correspondientes al nivel de ingresos y al puesto de trabajo son similares y considerablemente más bajas que las del nivel de estudios.

La asociación entre las valoraciones indirectas y el tipo de voz evaluada, el sexo y el colectivo del informante se presenta en la siguiente tabla.

\begin{tabular}{|l|l|l|l|}
\hline & $\begin{array}{l}\text { Media general } \\
\text { Valoraciones indirectas }\end{array}$ & $\mathrm{F}$ & $\mathrm{p}$ \\
\hline Voz masculina & 2,513 & 32,573 & 0,000 \\
\cline { 1 - 2 } Voz femenina & 2,969 & 3,995 & 0,047 \\
\cline { 1 - 2 } Hombre & 2,612 & \multirow{2}{*}{1,106} & 0,294 \\
\cline { 1 - 2 } & 2,787 & & \\
\hline Sin formación & 2,765 & 2,675 & \\
\hline Con formación & &
\end{tabular}

Tabla 6. Valoraciones indirectas según voz evaluada, sexo y colectivo

Se aprecia, nuevamente, que las medias de las valoraciones indirectas son más altas en el caso de la voz evaluada femenina, de las informantes mujeres y de los sujetos sin formación en variedades del español. La prueba de la varianza señala que se trata de diferencias con significación estadística solo en el caso del contraste del tipo de voz y del sexo de los sujetos. 


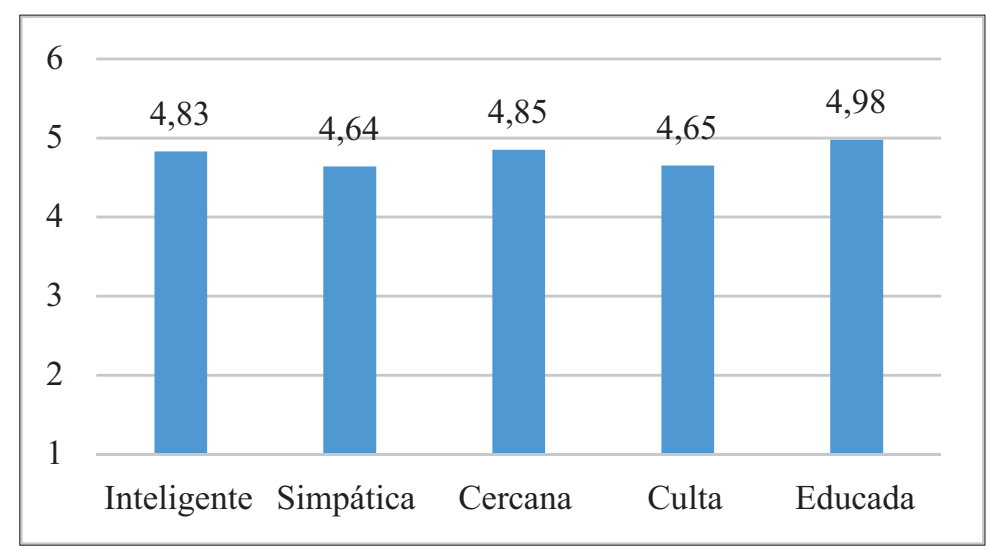

Gráfico 8. Medias de características personales: opiniones positivas y negativas

El gráfico 8 muestra que las medias para la valoración de la persona que habla son muy similares y se ubican en torno al 4,79, lo que demuestra que, en relación con la persona, la valoración realizada es altamente positiva. Algunas de las opiniones sobre las personas que hablan la variedad chilena son las siguientes: "amables y lindos; un poco sometidos" (651), "somos tímidos, fríos y distantes con desconocidos, pero al entrar en confianza muy extrovertidos" (654), "personas normales, comunes y corrientes" (664), "hablan entre dientes" (3601), "los chilenos somos gente simpática y amable, en su mayoría, aunque algo individualistas" (3605) y "hay de todo, gente agradable y gente muy desagradable" (325).

El cruce entre las valoraciones indirectas relativas a la persona que habla y la voz evaluada, el sexo y el colectivo de los sujetos se muestran, a continuación. Como puede apreciarse, el valor $\mathrm{p}$ de la tabla indica que no son valores estadísticamente significativos en ninguno de los tres casos, por lo tanto, solo se trata de antecedentes descriptivos.

\begin{tabular}{|c|c|c|c|}
\hline & $\begin{array}{c}\text { Media general } \\
\text { Características personales }\end{array}$ & $\mathrm{F}$ & $\mathrm{p}$ \\
\hline Voz masculina & 4,536 & \multirow[t]{2}{*}{2,052} & \multirow[t]{2}{*}{0,154} \\
\hline Voz femenina & 4,760 & & \\
\hline Hombre & 4,484 & \multirow[t]{2}{*}{2,381} & \multirow[t]{2}{*}{0,124} \\
\hline Mujer & 4,732 & & \\
\hline Sin formación & 4,709 & \multirow[t]{2}{*}{0,836} & \multirow[t]{2}{*}{0,362} \\
\hline Con formación & 4,566 & & \\
\hline
\end{tabular}

Tabla 7. Persona que habla según voz evaluada, sexo y colectivo 
Una vez más son la voz femenina, las mujeres de la muestra y los sujetos sin formación en variedades los factores que concitan las medias más altas.

En lo que sigue, se presentan los datos sobre las medias de las valoraciones indirectas de la variedad chilena a través de la región.

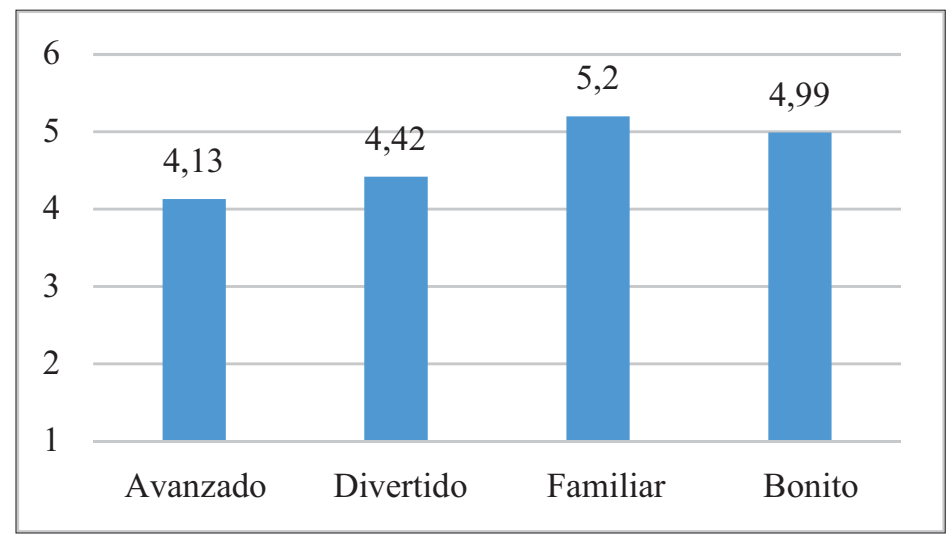

Gráfico 9. Valoraciones indirectas de la variedad chilena a través de la región

Los datos obtenidos de la valoración a través de la región o del país también permiten ver el valor positivo que se tiene de la propia variedad. De hecho, la media general es de 4,68, lo que constituye una de las medias más altas observadas en este estudio.

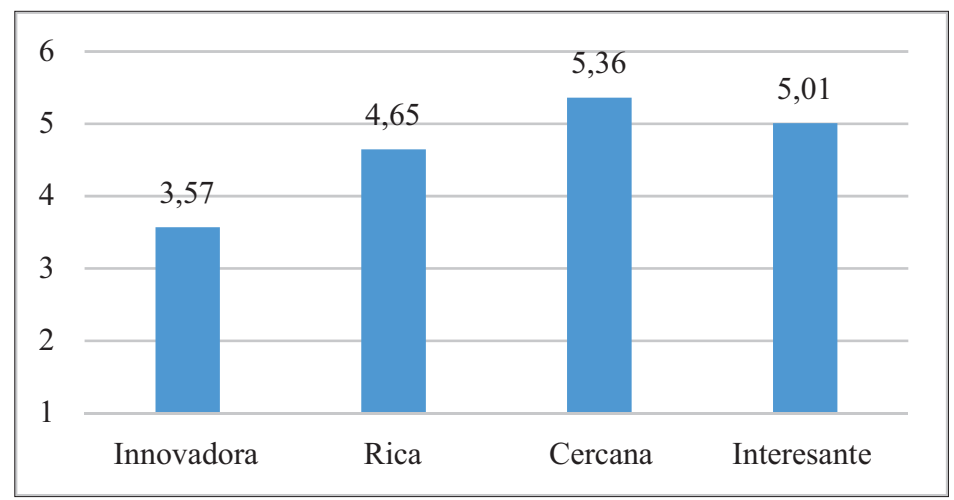

Gráfico 10. Valoraciones indirectas de la variedad chilena a través de la cultura

En lo que respecta a la valoración indirecta a través de la cultura, se puede observar nuevamente que se trata de un aspecto considerado como muy positivo, pues la media general es de 4,65. No obstante, a diferencia de lo 
que ocurrió con la valoración de la región, en la cultura el aspecto vinculado con la innovación (por oposición a lo tradicional) recibe una valoración que apenas sube de los 3,5 puntos, pero los altos promedios sobre lo cercana (opuesto a distante) e interesante (opuesto a poco interesante) de las voces evaluadas repercuten positivamente en el promedio general alcanzado.

Finalmente, la relación entre las valoraciones indirectas relativas a la región y la cultura y el tipo de voz evaluada, el sexo y el colectivo de los informantes se muestra en la tabla 8 . Se trata de datos no significativos, pero igualmente interesantes, porque permiten realizar contrastes con otras investigaciones.

\begin{tabular}{|c|c|c|c|c|c|c|}
\hline & $\begin{array}{c}\text { Media general } \\
\text { Región }\end{array}$ & $\mathrm{F}$ & $\mathrm{p}$ & $\begin{array}{l}\text { Media general } \\
\text { Cultura }\end{array}$ & $\mathrm{F}$ & $P$ \\
\hline Voz masculina & 4,521 & \multirow[t]{2}{*}{0,27} & \multirow[t]{2}{*}{0,870} & 4,498 & \multirow[t]{2}{*}{0,005} & \multirow[t]{2}{*}{0,945} \\
\hline Voz femenina & 4,547 & & & 4,508 & & \\
\hline Hombre & 4,461 & \multirow[t]{2}{*}{0,506} & \multirow[t]{2}{*}{0,478} & 4,451 & \multirow[t]{2}{*}{0,288} & \multirow[t]{2}{*}{0,592} \\
\hline Mujer & 4,577 & & & 4,534 & & \\
\hline Sin formación & 4,460 & \multirow[t]{2}{*}{0,846} & \multirow[t]{2}{*}{0,359} & 4,438 & \multirow[t]{2}{*}{0,742} & \multirow[t]{2}{*}{0,390} \\
\hline Con formación & 4,606 & & & 4,568 & & \\
\hline
\end{tabular}

Tabla 8. Región y cultura según voz evaluada, sexo y colectivo

Los promedios más altos se encuentran en la voz femenina, en las informantes mujeres y en los sujetos con formación en variedades tanto para la valoración de la región como de la cultura, aunque sin significación estadística.

\subsection{IDENTIFICACIÓN Y VALORACIÓN DEL RESTO DE LAS VARIEDADES CULTAS DEL ESPAÑOL}

A continuación, presentamos los hallazgos generales referentes al reconocimiento de las variedades del español de las voces evaluadas y a la percepción de los aspectos de sus características valorados positiva y negativamente por parte de los jóvenes universitarios chilenos de la muestra. Sin incluir la variedad chilena, se trata de 1400 valoraciones (100 informantes evaluando 14 voces cada uno). Hubo 734 (52,4\%) casos de identificación correcta de la variedad correspondiente, $593(42,3 \%)$ casos identificados incorrectamente y $72(5,3 \%)$ ocasiones en las que los informantes no respondieron o declararon no saber a qué variedad correspondía la voz 
evaluada. Como vemos, cuando se trata de identificar otras variedades del español, los informantes suelen confundirse, en especial, entre las variedades andaluza, canaria y caribeña. Presentamos a continuación el gráfico con el porcentaje de reconocimiento exacto del resto de las variedades cultas del español.

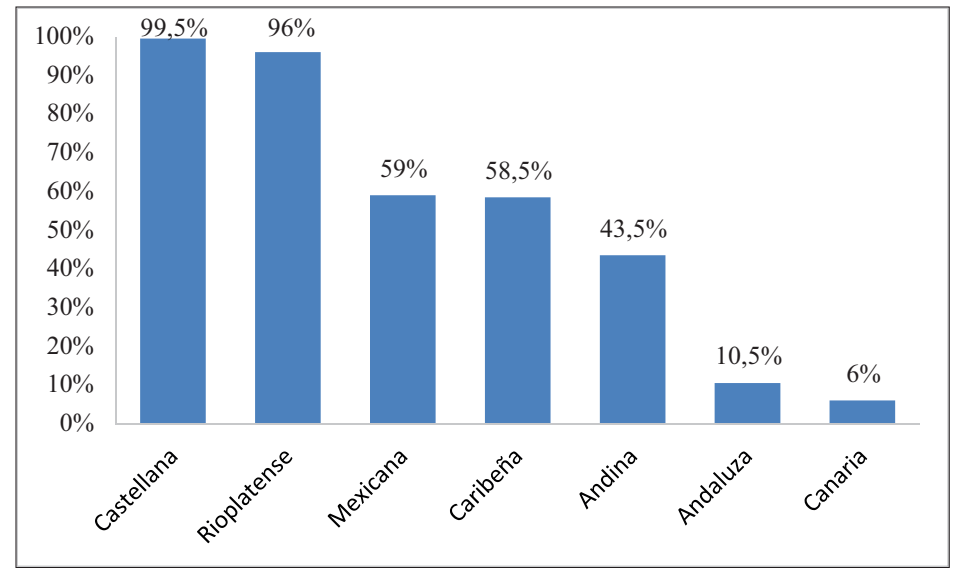

Gráfico 11. Porcentaje de identificación exacta de las variedades por universitarios jóvenes chilenos

La variedad más reconocible para los jóvenes universitarios chilenos es la variedad castellana, la que identifican de manera exacta en casi el total de casos valorados $(99,5 \%)$. Este dato es relevante, toda vez que se trata de una variedad que identifican con mayor exactitud que la variedad propia (ver tabla 2). Le sigue, en términos de porcentaje, la variedad rioplatense con un $96 \%$ de los casos identificados de manera exacta. Las frecuencias de identificación correcta disminuyen a algo más de la mitad de las voces evaluadas en la variedad mexicana $(59 \%)$ y en la caribeña $(58,5 \%)$, pero son las variedades andaluza y canaria las que tienden a no identificarse; la andaluza, porque suele identificarse como castellana y la canaria, porque se confunde con la variedad chilena. 


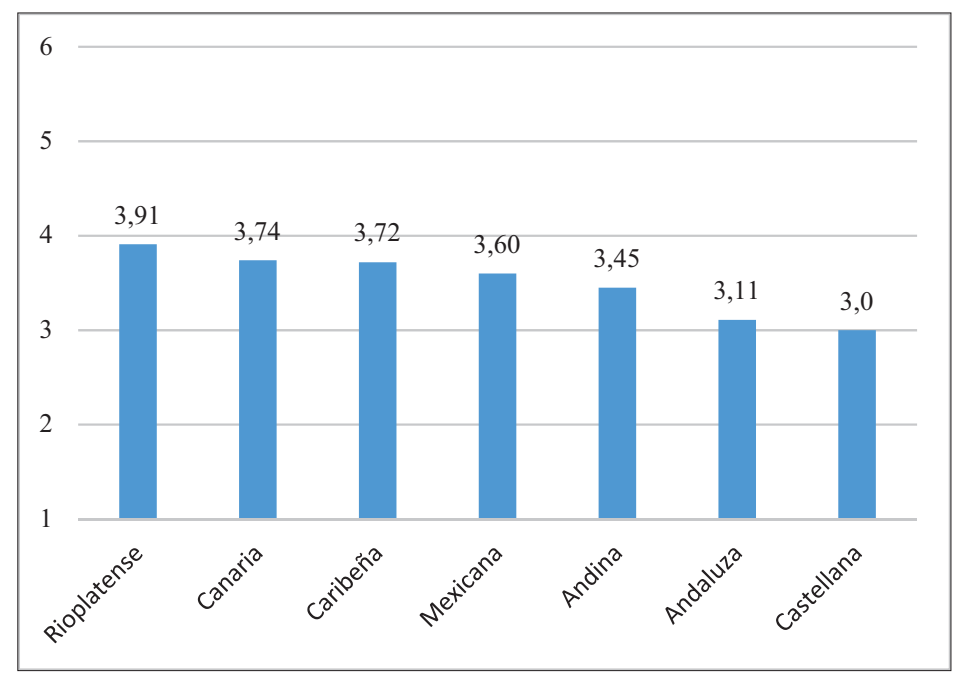

Gráfico 12. Valoración global directa de las variedades por universitarios jóvenes chilenos

La valoración directa global de las variedades cultas permite señalar que, en general, los dialectos que son identificados de manera correcta por los jóvenes universitarios chilenos no difieren mucho en su valoración, que, cabe destacar, tiende a ser positiva, en especial, cuando se trata de las variedades rioplatense, canaria y caribeña. Es relevante que las variedades caribeña y canaria fueron dos de las variedades más identificadas de manera incorrecta por los informantes, pero suelen valorarse de modo positivo, dada su cercanía $\mathrm{y}$, en especial, la canaria, debido a su parecido con la variedad propia (cf. tabla 2). Es interesante también, que el dialecto rioplatense no solo es el más reconocido por lo hablantes, sino que es también el que se ubica en el primer puesto de valoración directa.

En relación con los aspectos de la pronunciación valorados positivamente sin incluir la variedad chilena, se puede señalar que son los rasgos fónicos los que suelen identificarse de modo favorable con mayor frecuencia; se trata de 441/734 (60\%) casos en los que los informantes destacan aspectos como la fluidez, la claridad, la buena acentuación, el tono, la intensidad, el ritmo, la modulación, la suavidad, entre otros, como rasgos muy positivos de las variedades que evalúan. Le sigue, en orden de frecuencia, la opción de no contestar $(147 / 734,20 \%)$. A continuación, se ubica "ningún rasgo" $(68 / 734,9,3 \%)$ y otros $(66 / 734,9 \%)$, tales como la cercanía, la seguridad, lo cuidada, lo sexy, lo sencilla, lo familiar, lo bonita, lo divertida, lo agradable, lo segura, lo graciosa, lo simpática, lo urbana, lo calmada, lo culta, lo prolija, 
lo natural, lo espontánea y lo divertida de la voz evaluada. Con frecuencias bajas se identifican los rasgos pragmático-discursivos (10/734, 1,4\%), como el control de pausas, los marcadores del discurso y el énfasis, así como los rasgos léxicos $(2 / 734,0,3 \%)$ referidos a la amplitud léxica.

Sobre los aspectos de la pronunciación valorados de manera negativa por parte de los jóvenes universitarios chilenos, se puede concluir que, al igual que con respecto a los rasgos positivos, es el aspecto fónico el más destacado (390/734, 53,1\%), como la rapidez, la aspiración de la $s$ final de sílaba, la mala pronunciación, la escasez de acento, la lentitud, la pronunciación de $c h, y, l l, n$, la monotonía, el ritmo, la velocidad, el tono, la intensidad, la nasalidad, la agudeza y elisión de sonidos. "No contesta" es la segunda opción más preferida $(160 / 734,21,8 \%)$ seguida de "ninguno" (132/734, $18 \%)$. Continúan la categoría "otros" $(31 / 734,4,2 \%)$, relativa a lo distante, lo áspera, lo natural, lo aburrida, lo insegura, lo poco espontánea, lo seria y lo desagradable de la voz evaluada; los rasgos pragmático-discursivos (19/734, $2,6 \%$ ), como las muletillas, las vacilaciones, las pausas, los alargamientos y los titubeos; $y$, por último, los rasgos léxicos (2/734, 0,3\%), como el uso de tecnicismos relacionados con el ámbito laboral. Los informantes no reconocen ningún rasgo gramatical asociado a valoraciones positivas o negativas de las variedades evaluadas.

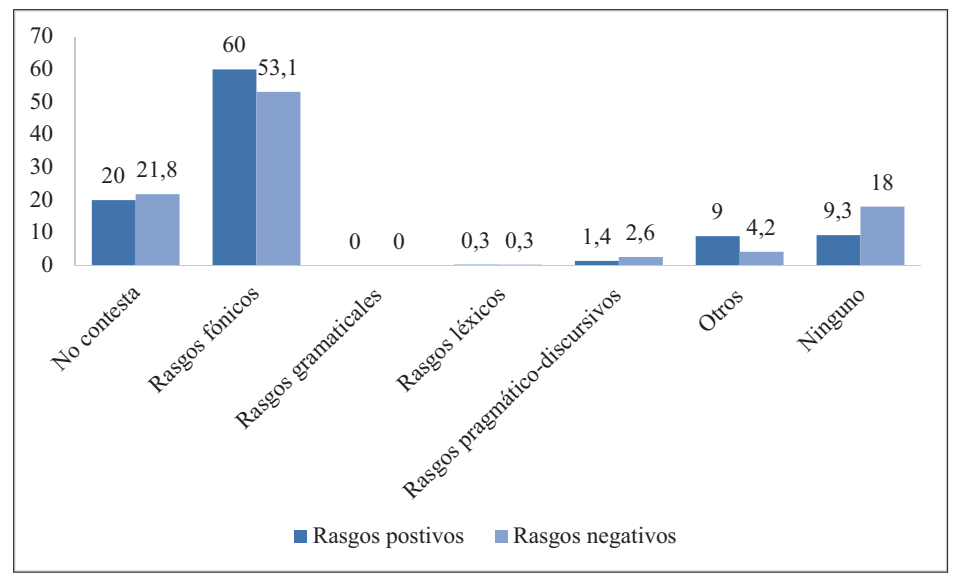

Gráfico 13. Comparación de los aspectos valorados positiva y negativamente de otras variedades del español (porcentajes)

Del gráfico precedente, se desprende que los jóvenes universitarios chilenos suelen reconocer los rasgos fónicos como un aspecto fundamental de las variedades cultas del español. El procentaje de rasgos de la pronunciación valorados positivamente es un poco mayor que el de los valorados 
negativamente, pero, en general, no se observan diferencias. En lo que respecta a "ningún" aspecto positivo o negativo, la valoración es más favorable para los aspectos positivos. Finalmente, se aprecia una pequeña diferencia en "otros", donde las características positivas superan, en porcentaje, a las negativas.

\section{CONCLUSIONES}

Las principales conclusiones de esta investigación son las siguientes:

1. La mayoría de los informantes considera que no existe una variedad que corresponda a un mejor español. Esto genera valoraciones donde se reconoce de manera explícita que se trata solo de variedades, donde no hay una mejor que otra. En un segundo orden, los informantes consideran como variedades en las que se habla mejor español a la variedad andina y la chilena, lo que implica una percepción muy positiva de la variedad propia.

2. Verificamos un alto nivel de acierto en el reconocimiento de la variedad propia, aunque no deja de ser interesante que la mayor parte de los casos de error en el reconocimiento de la variedad chilena corresponda a la variedad canaria.

3. Los aspectos fónicos son, asimismo, más reconocidos por los sujetos de la muestra, tanto para valoraciones positivas como negativas. Los informantes prácticamente no reconocen rasgos pragmáticodiscursivos ni léxicos para su propia variedad.

4. Sobre las valoraciones directas de la variedad chilena, se concluye que las dimensiones afectiva y cognitiva no presentan diferencias, pero destaca, en la dimensión afectiva, la cercanía de la variedad chilena. En cuanto a la dimensión cognitiva son destacables lo variada y lo suave del habla chilena. Con respecto a las variables consideradas en este estudio, se evalúa de mejor manera la voz femenina y evalúan más positivamente las mujeres. En particular, esta asociación contó con significación estadística en las valoraciones directas de la dimensión cognitiva y general y en las valoraciones indirectas.

5. En lo que respecta a las valoraciones indirectas de la variedad chilena, se observa que existen datos estadísticamente significativos para la 
valoración de la voz evaluada. Además, son significativos los datos relacionados con la variable sexo. Entre las características personales positivas, destaca la valoración sobre la educación de la persona que habla y, entre las características a través de la región y de la cultura, son destacables la familiaridad y la cercanía de las voces evaluadas.

6. Los hallazgos sobre las percepciones de las otras variedades del español nos permiten concluir, en términos generales, que los jóvenes universitarios chilenos suelen equivocarse al reconocer otras variedades, en especial, la canaria, la andaluza y la caribeña. Asimismo, se observa que suelen reconocer como positivos y negativos rasgos pertenecientes a la dimensión fonética de las variedades en estudio.

Por último, es necesario destacar el valor del instrumento utilizado para esta investigación. Además, ponemos de relieve que, con los datos previamente descritos, se contribuye al estudio de las actitudes lingüísticas de los hablantes chilenos con base en una metodología mixta que nos conduce hacia una aproximación más integral de este fenómeno.

\section{REFERENCIAS BIBLIOGRÁFICAS}

Blas Arroyo, José Luis. 1994. Valenciano y castellano. Actitudes lingüísticas de la sociedad valenciana. Estudio sobre una comunidad urbana. Hispania 77 (1): 143-155.

1999. Las actitudes hacia la variación intradialectal en la sociolingüística hispánica. Estudios Filológicos 34: 47-72.

2005. Sociolingüistica del español. Madrid: Cátedra.

Cestero, Ana María y Florentino Paredes. 2015a. Creencias y actitudes hacia las variedades normativas del español actual: primeros resultados del Proyecto PRECAVES XXI. Spanish in Context 12/2: 255-279.

2015b. Creencias y actitudes hacia las variedades del español en el siglo XXI: avance de un proyecto de investigación. En Dermeval da Hora, Juliene Lopes R. Pedrosa, Rubens M. Lucena (eds.). ALFAL 50 anos: contribuições para os estudos linguísticos e filológicos, pp. 652-683. João Pessoa: Ideia.

2018. Creencias y actitudes hacia las variedades cultas del español actual: el proyecto PRECAVES XXI. Boletín de Filología (este volumen).

García Marcos. 2015. Sociolingüistica. Madrid: Síntesis.

Labov, William. 1983. Modelos sociolingüísticos. Madrid: Cátedra.

López Morales, Humberto. 2015. Sociolingüística. Madrid: Gredos.

Moreno Fernández, Francisco. 2009a. Principios de sociolingüística y sociología del lenguaje. Barcelona: Ariel.

2009b. La lengua española en su geografia. Madrid: Arco Libros. 
Paredes, Florentino y Ana María Cestero. 2018. Percepciones de las variedades cultas del español por parte de hablantes del centro-norte de España según los datos del Proyecto PRECAVES XXI: el español ejemplar y la variedad propia. Oralia 21/1, pp. 87-112.

RoJAS, DARío. 2012a. Corrección idiomática atribuida al español de los países hispanohablantes por sujetos de Santiago de Chile. RLA. Revista de Lingüistica Teórica y Aplicada 50 (2): 39-62.

2012b. Actitudes lingüísticas en Santiago de Chile: agrado y variedades geográficas del español. Anuario de Lingüistica Hispánica XXVIII: 99-116.

2012c. Actitudes lingüísticas de hispanohablantes de Santiago de Chile: creencias sobre la corrección idiomática. Onomázein 26: 69-93.

2013. Estatus, solidaridad y representación social de las variedades de la lengua española entre hispanohablantes de Santiago de Chile. Literatura y Lingüística 29: 251270.

Silva-Corvalán, Carmen y Andrés EnriQue-Arias. 2017. Sociolingüística y pragmática del español. Washington, DC: Georgetown University Press.

Trudgill, Peter y Juan Manuel Hernández Campoy. 2007. Diccionario de sociolingüística. Madrid: Gredos. 